\title{
Effect Of Distributed Model Of Constraint Induced Movement Therapy For Subacute Stroke Patients
}

\author{
Muthuukaruppan Muthiah $^{1 *}$, Vikram Mohan ${ }^{2}$, Moses Arun Kumar ${ }^{3}$ \\ ${ }^{1,}$ School of Physiotherapy, Faculty of Allied Health Professions, AIMST University, Semeling, Kedah, Malaysia. \\ ${ }^{2}$ Department of Physiotherapy, Faculty of Health Sciences, Universiti Teknologi MARA, Puncak Alam, \\ Selangor, Malaysia. \\ ${ }^{3,}$ College of Physiotherapy, Meenakshi University, Virugambakkam, Chennai, India.
}

\begin{abstract}
Background: Constraint-induced movement therapy (CIMT) has proved to increase the amount and quality of function of an affected upper extremity after stroke by overcoming learned non-use to bring about functional reorganization of the primary motor cortex. The objective of the study was to examine the effects of distributed model of CIMT in improving upper extremity (UE) functions in subacute stroke and to study the importance of constraint in improving the upper extremity function.
\end{abstract}

Methods: Sixteen subjects with subacute stroke were recruited based on the inclusion and exclusion criteria. Subjects were assigned to the experimental (constraint) group and the control (non-constraint) group using random sampling method. Subjects in the constraint \& the non-constraint group were provided therapy for 3 hours with repetitive functional task practice. The subjects in the constraint group wore the constraint for $5 \mathrm{hrs} /$ day on their less affected UE which included $2 \mathrm{hrs}$ at home and $3 \mathrm{hrs}$ during repetitive functional task practice for 20days. The non-constraint group did not wear the constraint. Three UE subscales of the motor assessment scale were used to measure the activity level of the more affected arm pretest \& posttest.

Results: The results expressed that the constraint group significantly improved with $P=0.008(P<0.01)$ than the non-constraint group, which emphasizes that distributed model of constraint induced movement therapy could facilitate the UE function after stroke in subacute patients.

Conclusion: The constraint group significantly improved than the non-constraint group, which emphasizes that distributed model of CIMT could improve the upper extremity function after stroke in subacute patients

Keywords: Constraint induced movement therapy, upper extremity function, subacute stroke and repetitive functional task practice

\section{Introduction}

Upper extremity (UE) dysfunction among stroke population reduces patient's independence and has an impact on activities of daily living and quality of life. ${ }^{1}$ Muscle weakness and loss of dexterity are important factors for the reduced upper extremity function in stroke patients. ${ }^{2}$ Activities such as grasping, holding, and manipulating objects are daily functions of upper extremity, lacking in $55 \%$ to $75 \%$ of patients with 3 to 6 months following stroke. ${ }^{3}$ Hence, strategies to improve upper extremity function among stroke population are necessitated to ameliorate motor recovery as motor recovery will decelerate in subacute ( $>3$ months) and chronic ( $>1$ year) stroke phases. ${ }^{4,5}$ Traditional neurophysiological approaches developed by Bobath, Brunstrom, Rood \& Kabat for enhancing recovery in stroke rehabilitation, lack to date scientific evidence. ${ }^{6,7}$ Research in facilitating neuroplasticity led to the development of new movement therapy protocols inducing crucial neural and motor recovery. Movement therapy protocols like the task specific training, CIMT, and mental imagery have convincing evidence for their role in neural reorganization and associated motor and functional recovery. ${ }^{8,9,10}$ Movement therapy protocols based on motor learning principles are capable of facilitating neural reorganization post stroke. ${ }^{11,12}$

Motor learning refers to permanent changes in behavior that occurs due to practice \& experience. ${ }^{13}$ Movement therapy protocols target deficits in the neuromuscular system and use repetition or an experience for improving skilled motor activity. ${ }^{14}$ Repetitive practice for reaching to a glass of water improves the elbow extension, causes structural and functional changes in the motor cortex and cerebellum. Such changes are indicative of motor recovery, which is permanent. ${ }^{15}$ Changes are not found with simple exercises, such as performing elbow flexion extension without any goal. ${ }^{16}$ These findings aided in developing upper extremity rehabilitation protocols for stroke with increasing exercise duration and intensity ${ }^{17,18,19}$ focusing on task-specific training. ${ }^{20}$ 
Task-specific training with or without constraining the less affected UE had resulted in significant performance of the more affected UE in both chronic and subacute stroke survivors. ${ }^{19,20,21}$ Utilizing the above said facts, constraint-induced movement therapy (CIMT) emphasizes to increase the amount and quality of the more affected UE function after stroke by using the three components: constraint over the less affected UE, intensive practice to the more affected UE (massed practice) and reinforcement of successive attempts with the more affected UE (shaping). ${ }^{22,23}$ The rationale behind CIMT is to overcome learned non-use and to bring about functional reorganization of the primary motor cortex.

Traditional model of CIMT on humans were conducted involving training of the affected upper limb (6 hours a day, week-days for 2 weeks) and constraint to the less affected upper extremity (90\% of the waking hours for 14 consecutive days) after successfully conducting it on non-human primates. ${ }^{24,25}$ Despite the positive outcomes of the traditional model, $68 \%$ of patients with CVA refused to participate in the traditional model and $80 \%$ patients felt, if CIMT protocol lasts for more than 2 weeks with shorter PT/OT sessions and/or fewer hours wearing the constraints, they would participate. ${ }^{26}$ In association to all these drawbacks, Page and colleagues introduced the modified constraint-induced movement therapy featuring half hour physical and occupational therapy sessions 3 times / week for 10 weeks, wearing of constraint on the unaffected upper limb for 5 hours for 5 days per week and demonstrated significant results. ${ }^{26,27,28,29}$

Distributed CIMT model encompasses $60 \mathrm{hrs}$ of therapy as the traditional model but staggered in 3 weeks. The restraint is worn similar to the modified model for $5 \mathrm{hrs} /$ day. ${ }^{30}$ Page et al have proved that schedules of reduced amount and intensity of CIMT can improve function in upper extremity but have not evaluated the effect of only manipulating the training intensity. ${ }^{30}$

There is no published study on CIMT in the region and distributed model of CIMT has not been analyzed extensively. CIMT studies on subacute stroke patients are very less and distributed model of CIMT on subacute stroke patients are negligible. The purpose of this study was to examine the effects of distributed model of CIMT in improving upper extremity functions in subacute stroke patients and to study the importance of constraint in improving the upper extremity function in distributed model of CIMT.

\section{Materials And Methods}

\section{Design}

Experimental Pretest - Posttest control group design was used with a cohort of subjects with subacute stroke $\left(3-12\right.$ months after onset of stroke). ${ }^{31,32}$ This study was completed within a period of 9 months. 16 subjects selected for the study were randomized to either of the two groups - CIMT (Constraint) group and Control (Nonconstraint) group using the computer generated list of consecutive random numbers. Meenakshi university ethical review board approval was obtained before the commencement of the study. Informed consent was obtained from all the participants.

\section{Subjects}

Subjects were volunteers recruited from the out-patient department of Meenakshi college of physiotherapy. Potential subjects were screened to determine if they met the following inclusion criterias: Sub acute stroke patients ${ }^{27,32}$, Score of 19 or more on Mini mental state exam ${ }^{27}, 20^{\circ}$ of wrist extension \& $10^{\circ}$ of finger extension in affected upper extremity ${ }^{32}$, Score of 2 or more in the "Upper arm function" section but less than 2 in the "Advanced hand activities" section on the Motor assessment scale ${ }^{27}$. Subjects were excluded if they exhibited: Excessive spasticity $\geq 3$ on the Modified Ashworth Scale, Excessive pain in the affected upper limb, as measured by a score of $\geq 4$ on a 10 point visual analog scale, Patients participating in any clinical trial. 16 patients were selected using the above mentioned criterias. Constraint group had 8 subjects, mean age $48.5+3.4 y r s$ (39-57 yrs); 1 female \& 7 males; 6 right hemiparesis and 2 left hemiparesis. Non-constraint group had $\overline{8}$ subjects mean age $52.5+3.1 \mathrm{yrs}(44-59 \mathrm{yrs})$; 1 female $\& 7$ males; 5 left hemiparesis and 3 right hemiparesis (Table 1). Patients and their caregivers were informed of the training's basic principles, including the expected disappointment during the first several days after the forced non-use of the unaffected upper extremity.

\section{Protocol}

All subjects selected for the study received $3 \mathrm{hrs}$ of repetitive functional task practice to their more affected upper extremity for 3 weeks. The constraint group wore the constraint for 5 hours a day ${ }^{29}$ on the less affected upper extremity while the non-constraint group did not wear constraint. Constraint is a cotton upper arm sling, with a strap around the neck for support. Constraint was applied for 3 hours during the training sessions and another 2 hours at home. Subjects were not asked to wear the constraint in unstable environments and during bilateral hand activities (e.g. opening bottles, lifting jars). The subjects were encouraged to wear the constraint independently; subjects who could not wear it independently wore it with assistance. The subjects were provided with a log book to note the restraint use, to be filled by the caregiver and list the activities performed during the constraint wear period. This log would be reviewed every day before the therapy session, 
for the duration of constraint applied and to assess the amount of arm used during those periods. Subjects in the non-constraint group were also given therapy for 3 hours with the same repetitive functional task practice but without constraining the unaffected arm, which didn't restrict the subjects in occasionally using the unaffected arm to assist their affected arm in activities. The functional task practice includes: picking up glass of water \& drinking, reaching for an item and putting into the mouth, opening lid of bottles, arranging puzzles, peg boards and in real home environment the activities encouraged were turning handles of doors, turning pages of newspapers, magazines.

\section{Intervention}

Subjects were made to repeatedly attempt to use the more affected UE for activities, assisted by the therapist whenever essential. This assisted in achieving Massed Practice. Tasks were selected to address the motor deficits of the individual subjects. The tasks if complex for the subject were broken down into simple components and then made to perform together (Shaping). During the therapy sessions, the therapists enhanced to focus participants attention and effort to the use of the affected upper extremity. Every task was repeated 610 times with the more affected UE. The levels of the tasks are adjusted so that as the subject improves, there is increase in the challenge of the task. (e.g.) facilitating grasping and release of the affected hand, initially larger tennis ball was used as the subjects improved smaller balls were used to facilitate grasping and release. The therapist worked with subjects on 1:1 therapist to subject ratio. The constraint is worn for $2 \mathrm{hrs}$ at home and during $3 \mathrm{hr}$ repetitive functional task practice sessions. The subjects were monitored through their caregivers on their $1 \mathrm{hr}$ home program schedule during which the constraint group had to wear the constraint. The constraint was worn for another $1 \mathrm{hr}$ at home other than home program session, which was identified as the frequent arm use period at home, thus encouraging use of more affected UE at real life situations. Posttest measurements were performed thrice at the end of each week to measure the difference in the activity level of the more affected upper extremity. The examiner was blinded and unaware of the intervention the subjects had undergone.

\section{Outcome Measures}

Pretest was performed to measure the baseline characteristics before the study started and the subjects were blinded of their group. Baseline measurement of all subjects both in constraint and non - constraint group were scored on the three UE sub scales of motor assessment scale (Upper arm functions, Hand movements \& Advanced hand activities). Motor assessment scale is a 6 point ordinal scale, measuring the activity level of the more affected UE after stroke. Motor assessment scale has shown test-retest reliability $(\mathrm{r}=0.98)$, inter-rater reliability $(\mathrm{r}=0.95) \cdot{ }^{33}$ Motor assessment scale responses are similar to the responses of Action reach arm test which is one of the common outcome measures used in CIMT studies in measuring upper extremity functions after stroke. ${ }^{34}$

\section{Result}

The characteristics of the study subject were as follows: Stroke survivors were $4.4-10.5$ months $(7.10$ months \pm 2.03$)$ post onset of stroke for the constraint group, and $4.5-9.3$ months ( 7.45 months \pm 1.83$)$ post onset of stroke for the non-constraint group (Table 1). Baseline characteristics showed no significant difference between the constraint and non-constraint groups before starting the study. Motor assessment scale was used to record the changes in the more affected UE activity in both the groups; the data collected not being normally distributed and hence subjected to non-parametric statistical evaluation on the SPSS version 17.0.

TABLE 1: Physical Characteristics of study subjects

\begin{tabular}{|l|l|l|}
\hline Physical Characteristics & $\begin{array}{l}\text { Constraint group } \\
\text { (Experimental) }\end{array}$ & Non-constraint group (Control) \\
\hline Number of subjects & 8 & 8 \\
$(1 \mathrm{Male}, 7$ Female) & $(1 \mathrm{Male}, 7 \mathrm{Female})$ \\
\hline Age, mean(SD), range (years) & $48.5(3.4),(39-57)$ & $52.5(3.1),(44-59)$ \\
\hline Right Hemiparesis & 6 & 5 \\
Left Hemiparesis & 2 & 3 \\
\hline Duration of stroke (months) & $7.1(2.03)$ & $7.45(1.83)$ \\
$-\quad$ Mean (SD) & 6.7 & 7.9 \\
\hline$\quad$ Median (range) & & \\
\hline
\end{tabular}

Wilcoxon's signed rank test was used to compare the pre and post test $3^{\text {rd }}$ week values for the constraint $\&$ non-constraint group. Mean, Median, Standard deviation, $\mathrm{P}$ value $\& \mathrm{Z}$ value for both groups are given in (Table 2). The comparison of Pretest $\&$ Posttest $3^{\text {rd }}$ week values showed there were significant differences in the upper extremity function when compared with the non-constraint group. Both groups exhibit a significance of (P $<0.01$ ), supporting the experimental hypothesis. 
TABLE 2: Comparison of Pre and Post upper extremity recovery among Non-Constraint and Constraint group

\begin{tabular}{|l|l|l|l|l|l|l|l|}
\hline \multicolumn{2}{|l|}{} & Pre-test & $\begin{array}{l}\text { Post-test } \\
\text { week }\end{array}$ & $\begin{array}{l}\text { Post-test 2 } \\
\text { week }\end{array}$ & $\begin{array}{l}\text { Post-test 3 } \\
\text { week }\end{array}$ & Z Value & P Value \\
\hline $\begin{array}{l}\text { Control group } \\
\text { (Non-Constraint) }\end{array}$ & $\begin{array}{l}\text { Mean \& S.D } \\
\text { Median }\end{array}$ & $\begin{array}{l}7.5 \pm 2.27 \\
7.5\end{array}$ & $\begin{array}{l}8.0 \pm 2.00 \\
8.0\end{array}$ & $\begin{array}{l}8.63 \pm 2.07 \\
8.5\end{array}$ & $\begin{array}{l}9.13 \pm 2.23 \\
9.0\end{array}$ & 2.598 & 0.009 \\
\hline $\begin{array}{l}\text { Experimental group } \\
\text { (Constraint) }\end{array}$ & $\begin{array}{l}\text { Mean \& S.D } \\
\text { Median }\end{array}$ & $\begin{array}{l}8.0 \pm 2.62 \\
8.0\end{array}$ & $\begin{array}{l}8.0 \pm 2.62 \\
7.5\end{array}$ & $\begin{array}{l}9.0 \pm 3.07 \\
8.5\end{array}$ & $\begin{array}{l}10.9 \pm 2.6 \\
10.5\end{array}$ & 2.636 & 0.008 \\
\hline
\end{tabular}

The constraint group has clinically improved in its upper extremity function by $40 \%$, the non-constraint group has improved by $20 \%$, which is considered two fold than the non-constraint group. This is calculated from the median values of both groups (Table 3) \& (Graph 1). The constraint group has clinically improved in upper arm function subscale by $22 \%$ when compared to $0 \%$ in non-constraint group in three weeks, hand movements have improved $100 \%$ in constraint group when compared to $50 \%$ in non-constraint group. The advanced hand activities have not improved in both the groups. According to the logs, 14 out of 16 patients reported successful accomplishment using constraints for 5 hours a day. The remaining 2 patients had some difficulties in compliance and used the constraint for $80 \%$ of the scheduled constraint wear period.

TABLE 3: Comparison of Pre and Post median values of upper extremity recovery on Motor assessment scale among Constraint and Non-Constraint group

\begin{tabular}{|l|l|l|}
\hline & Non-Constraint group & Constraint group \\
\hline Pre test & 7.5 & 7.5 \\
\hline Post test $3^{\text {rd }}$ week & 8.5 & 10.5 \\
\hline Percentage of Recovery & 20 & 40 \\
\hline
\end{tabular}

FIGURE 1: Comparison of upper extremity recovery among Non-Constraint and Constraint group

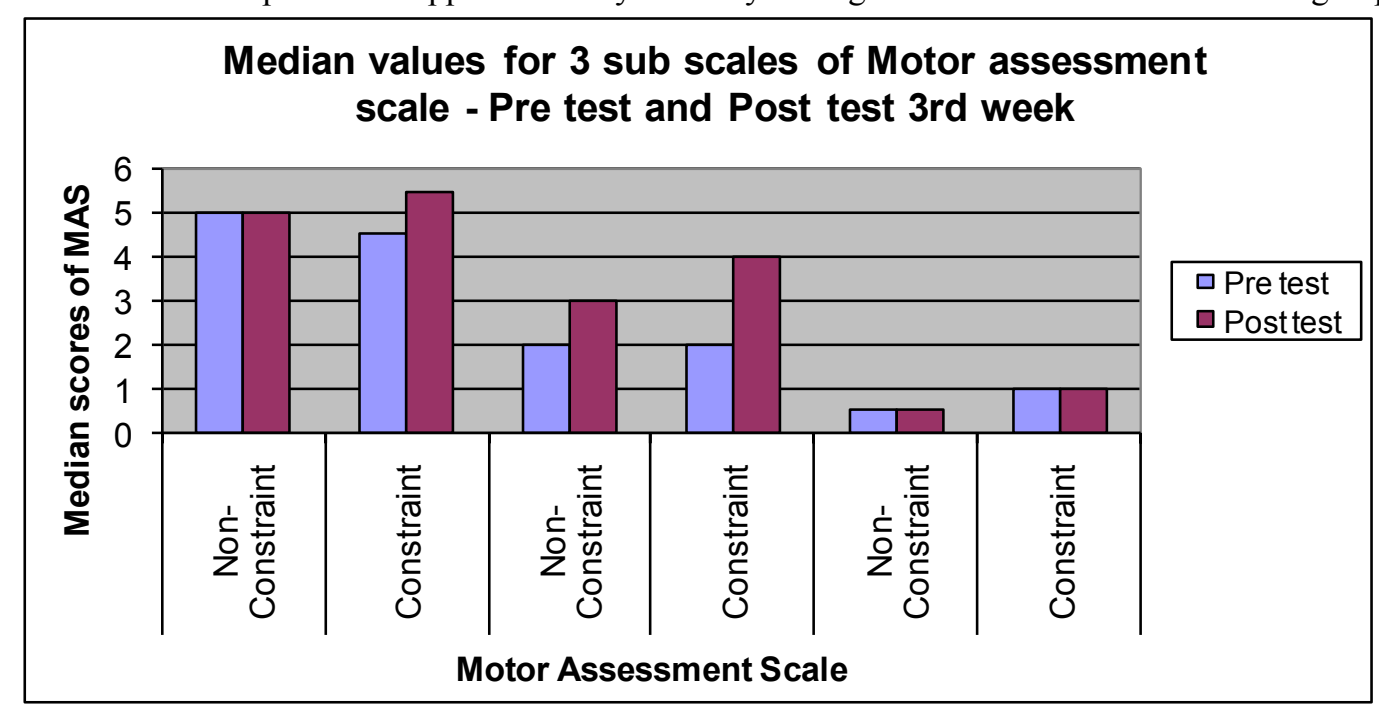

IV. Discussion

Distributed model of CIMT is the latest model of the family, with different schedule of therapy and restraint protocol. Traditional CIMT models engaged for 60 hours of therapy, for a span of 14 days and constraint application for $90 \%$ of the waking hours every day. ${ }^{23}$ Modified CIMT includes 60 hours of therapy for a span of 10 weeks with constraint applied for 5 hours every day. ${ }^{26}$ The distributed model has therapy schedule for 60 hours in 20 days and constraint applied for 5 hours every day. ${ }^{30}$ Systematically implementing 6 hours therapy per day similar to the traditional model in the Asian circumstance is practically impossible due to the patient turn over and sustainability. It has been identified that the intensity of training is an important factor in CIMT intervention, thus we stuck to $60 \mathrm{hrs}$ of therapy even though staggered for 3 weeks. ${ }^{23,35,36}$ The present study results indicated that distributed model of CIMT for 3 weeks promoted UE function following subacute stroke. The findings of the current study are consistent with those carried out in American population..$^{23,27,28}$ Similarly present study result agrees with other studies that have evaluated the effect of forced use. ${ }^{34}$ Maintaining the activities common for both experimental and control group this study was able to demonstrate that application of constraint improves the affected upper extremity function in contrast to the previous research. ${ }^{37}$ The positive outcome of our study can be explained to the commitment of subjects \& caregivers in complying with the constraint wear schedule of $5 \mathrm{hrs}$ a day. 
Since our study is one such research which had utilized motor assessment scale, from the study output, we would like to recommend motor assessment scale could be replaced with action research arm test in future CIMT studies. However previous studies highlighted at 2 years follow-up, the effects were higher in the group that had been constrained. ${ }^{38}$ Possible limitation of the study was not evaluating the effect of constraint and distributed model of CIMT over the impairment and functional domains post stroke. The effect and compliance of constraint application was not recorded objectively. Therefore future studies should involve the measurement of distributed model of CIMT on impairment and functional domains and use an objective tool to observe the effect and compliance of constraint use.

\section{Conclusion}

The results of the study showed that the constraint group significantly improved than the non-constraint group, which emphasizes that distributed model of CIMT could improve the upper extremity function after stroke in subacute patients. This study also emphasized the significance of constraint in distributed model of CIMT.

\section{Acknowledgements:}

We thank the subjects and their caregivers for their participation and support to this study.

\section{References}

[1] Page SJ, Sisto SA, Levine P, McGrath RE. Efficacy of modified constraint-induced movement therapy in chronic stroke: A singleblinded randomized controlled trial. Archives of Physical Medicine and Rehabilitation 2004; 85: 14-18.

[2] Bakheit AMO, Thilmann AF, Ward AB, Poewe W, Wissel et al. A Randomized, Double-Blind, Placebo-Controlled, Dose-Ranging study to compare the efficacy and safety of three doses of botulinum toxin type A (Dysport) with placebo in upper limb spasticity after stroke. Stroke 2000; 31:2402-2406.

[3] Gad Alon, Alan F. Levitt and Patricia A. McCarthy. Functional electrical stimulation enhancement of upper extremity functional recovery during stroke rehabilitation: A Pilot Study. Neurorehabilitation Neural Repair 2007; 21: 207-215.

[4] Jorgenson HS, Nakayama H, Raaschou H, et al. Outcome and time course of recovery in stroke, part II: time course of recovery. The Copenhangen Stroke Study. Arch Phys Med Rehabil. 1995; 76: 406-412.

[5] Siegert RJ, Lord S, \& Porter K. Constraint-induced movement therapy: Time for a little restraint. Clinical Rehabilitation 2004; 18 : 110-114.

[6] Paci, M. Physiotherapy based on the Bobath concept for adults with post-stroke hemiplegia: A review of effectiveness studies. Journal of Rehabilitation Medicine 2003; 35 (1), 2-7.

[7] Hafsteinsdo'ttir TB, Algra A, Kappelle LJ, Grypdonck MH, Dutch NDT Study Group. Neurodevelopmental treatment after stroke: a comparative study. Journal of Neurology Neurosurgey Psychiatry 2005; 76 (6), 788-792.

[8] Wolf SL, Winstein CJ, Miller JP et al. Effect of constraint-induced movement therapy on upper extremity function 3 to 9 months after stroke: the EXCITE randomized clinical trial. JAMA 2006; 296: 2095-2014

[9] Page SJ, Levine P, Leonard A. Mental practice in chronic stroke: results of a randomized, placebo-controlled trial. Stroke 2007a; 38: 1293-1297.

[10] Gauthier LV, Taub E, Perkins C, Ortmann M, Mark VW et al. Remodeling the brain: plastic structural brain changes produced by different motor therapies after stroke. Stroke 2008; 39 (5): 1520-1525.

[11] Jones TA, Allred RP, Adkins DL, Hsu JE, O'Bryant A et al. Remodeling the brain with behavioral experience after stroke. Stroke 2009; 40 (3 Suppl.): S136-S138.

[12] Krakauer JW. Motor learning: its relevance to stroke recovery and neurorehabilitation. Current Opinion Neurology 2006; Feb. 19 (1): 84-90.

[13] Schmidt RA. Motor control and learning: a behavioral emphasis, Human Kinetics 2005; $4^{\text {th }}$ edition: 1-22.

[14] Jette DU, Latham NK, Smout RJ, Gassaway J, Slavin MD, Horn SD. Physical therapy interventions for patients with stroke in inpatient rehabilitation facilities. Physical Therapy 2005; 85 (3): 238-248.

[15] Kleim JA, Jones TA. Principles of experience-dependent neural plasticity: implications for rehabilitation after brain damage. Journal of Speech Language \& Hearing Research 2008; vol. 51: S225-S239.

[16] Nudo RJ, Plautz EJ, Frost SB. Role of adaptive plasticity in recovery of function after damage to motor cortex. Muscle Nerve 2001; 24 (8): 1000-1019.

[17] Kwakkel G, Wagenaar RC. Effect of duration of upper- and lower-extremity rehabilitation sessions and walking speed on recovery of interlimb coordination in hemiplegic gait. Physical Therapy 2002; 82(5): 432-448.

[18] Duncan P, Studenski S, Richards L, et al. Randomized clinical trial of therapeutic exercise in subacute stroke. Stroke 2003; 34(9): 2173-2180.

[19] Feys H, De Weerdt W, Verbeke G, et al. Early and repetitive stimulation of the arm can substantially improve the long-term outcome after stroke: a 5-year follow-up study of a randomized trial. Stroke 2004; 35(4): 924-929.

[20] Winstein CJ, Rose DK, Tan SM, Lewthwaite R et al. A randomized controlled comparison of upper-extremity rehabilitation strategies in acute stroke: a pilot study of immediate and longterm outcomes. Arch of Phys Med Rehabil. 2004; 85: 620-628.

[21] Kwakkel G. Impact of intensity of practice after stroke: issues for consideration. Disability Rehabilitation 2006; 28: 823-830.

[22] Taub E, Miller NE, Novack TA, Cook IE, Fleming WC, Nepomuceno CS et al. Technique to improve chronic deficit after stroke. Arch of Phys Med and Rehabil 1993; 74: 347-354.

[23] Taub E, Uswatte G, Mark VW, \& Morris DM. The learned nonuse phenomenon: implications for rehabilitation. Europa Medicophysica 2006a; 42: 241-256.

[24] Taub E, Uswatte G, King DK, Morris D, Crago JE et al. A placebo-controlled trial of constraint induced movement therapy for upper extremity after stroke. Stroke 2006b; 37: 1045 - 1049.

[25] Page SJ, Sisto SA, Levine P, Johnston MV et al. Modified constraint induced therapy: a randomized, feasibility and efficacy study. Journal of Rehabilitation Research and Development 2001; 38: 583-590. 
[26] Page SJ, Sisto SA, Johnston MV et al. Modified constraint induced therapy in subacute stroke: a case study. Arch of Phys Med Rehabil. 2002a; 83: 286-290.

[27] Page SJ, Sisto SA, Levine P. Modified constraint-induced therapy in chronic stroke. Am J Phys Med Rehabil. 2002b; 81: 870-875.

[28] Page SJ \& Levine P. Modified constraint-induced movement therapy in patients chronic stroke exhibiting minimal movement with ability in the affected arm. Physical Therapy 2007b; 87: 872-878.

[29] Dettmers C, Teske U, Hanzei F, Uswatte G \& Taub E. Distributed form of constraint - induced movement therapy improves functional outcome and quality of life after stroke. Archives of Physical Medicine and Rehabilitation 2005; vol. 86: $204-209$.

[30] Blanton S, Wolf SL. An application of upper extremity constraint - induced movement therapy in a patient with subacute stroke. Physical Therapy 1999; Vol. 79: No.9.: 847-853.

[31] Chae J, Bethoux F, Bohine T, et al. Neuromuscular stimulation for upper extremity motor and functional recovery in acute hemiplegia. Stroke 1998; 29(5): 975-979.

[32] Morris D, \& Taub E. Constraint-induced therapy approach to restoring function after neurological injury. Topics in Stroke Rehabilitation 2001; 8: 16-30.

[33] Malouin F, Pichard L, Bonneau C, Durand A, Corriveau D. Evaluating motor recovery early after stroke: comparison of the FuglMeyer assessment and the motor assessment scale. Arch of Phys Med Rehabil. 1994; 75(11): 1206-1212.

[34] Taub E, Uswatte G, Pidikiti R. Constraint-induced movement therapy: a new family of techniques with broad application to physical rehabilitation - a clinical review. Journal of Rehabil Res Deve 1999; 36: 237-251.

[35] Page SJ, Levine P, Leonard AC. Modified constraint induced therapy in acute stroke: A randomized controlled pilot study. Neurorehabilitation Neural Repair 2005; 19: 27-32.

[36] Hashem AT, Abdul-Majeed A, Ziad S, Saleh A-O. Constraint induced movement therapy for stroke survivors in Jordan: a homebased model. International Journal of Therapy and Rehabilitation 2010; vol. 17: No. 12: 638-645.

[37] Carr JH, Shepherd RB, Nordholm L, Lynne D. Investigation of a new motor assessment scale for stroke patients. Phys Ther 1985; 65:175-180.

[38] Van der Lee JH, Snels IAK, Beckerman H, Lankhorst GJ et al. Exercise therapy for arm function in stroke patients: a systematic review of randomized controlled trials. Clinical Rehabilitation 2001; 15: 20-31. 